Research Paper

\title{
Effect of IL-18 on the Expansion and Phenotype of Human Natural Killer Cells: Application to Cancer Immunotherapy
}

\author{
Hiroaki Senju1,2,3, Asuka Kumagai ${ }^{2}$, Yoichi Nakamura ${ }^{1,3}$, Hiroyuki Yamaguchi1 ${ }^{1,3}$, Katsumi Nakatomi1 ${ }^{1,3}$, \\ Shota Fukami², Kengo Shiraishi², Yuka Harada², Mitsuhiro Nakamura², Haruki Okamura4, Yoshimasa \\ Tanaka $^{2,4}$ and Hiroshi Mukae ${ }^{1,3}$ \\ 1. Department of Respiratory Medicine, Graduate School of Biomedical Sciences, Nagasaki University, 1-7-1 Sakamoto, Nagasaki 852-8501, Japan \\ 2. Center for Bioinformatics and Molecular Medicine, Graduate School of Biomedical Sciences, Nagasaki University, 1-12-4 Sakamoto, Nagasaki 852-8523, \\ Japan \\ 3. Second Department of Internal Medicine, Nagasaki University School of Medicine, 1-7-1 Sakamoto, Nagasaki 852-8501, Japan \\ 4. Hyogo College of Medicine, 1-1 Mukogawa, Nishinomiya, Hyogo 663-8501, Japan \\ $\triangle$ Corresponding author: ystanaka@nagasaki-u.ac.jp, phone number: (81)-958198522
}

(c) Ivyspring International Publisher. This is an open access article distributed under the terms of the Creative Commons Attribution (CC BY-NC) license (https://creativecommons.org/licenses/by-nc/4.0/). See http://ivyspring.com/terms for full terms and conditions.

Received: 2017.09.13; Accepted: 2018.02.13; Published: 2018.03.09

\begin{abstract}
When pathogenic stresses are recognized by innate immune cells, inflammasomes are assembled and caspase- 1 is activated, resulting in the conversion of pro-IL-18 into mature IL-18. Because natural killer (NK) cells express IL-18 receptors, IL-18 may play roles in immune functions of NK cells. In the present study, we examined the effect of IL-18 on NK cells derived from lung cancer patients and healthy adult volunteers. When peripheral blood NK cells were stimulated with IL-2, the cells formed clusters beginning on day 5-6 and proliferated thereafter, in which the number of NK cells increased by 10-fold in 10 days. When IL- 18 was added, cell clusters were observed as early as on day 4 and NK cells proliferated vigorously. On day 10 , the expansion rate was 56 -fold on average, showing that IL-18 promoted the expansion of NK cells. It was also notable that IL-18 enhanced the expression of CD80, CD86, HLA-DR and HLA-DQ on NK cells, suggesting that IL-18 conferred NK cells an APC-like phenotype. When cellular cytotoxicity was determined, APC-like NK cells efficiently killed tumor cells and anti-tumor activity was augmented by the addition of tumor antigen-specific mAbs. In addition, IFN- $\gamma$ was produced by APC-like NK cells in response to tumor cells, and the cytokine production was further enhanced by mAbs. Taken together, IL-18 not only promoted the expansion of NK cells, but also changed the phenotype of NK cells. IL-2/IL-18-induced NK cells might, therefore, serve as a bridge between innate immunity and adaptive immunity and be useful for cancer immunotherapy.
\end{abstract}

Key words: IL-18, NK cells, antigen-presenting cells, PD-1, immune checkpoint

\section{Introduction}

Cancer immunotherapy has become the standard treatment modality after immune checkpoint inhibitors were shown to have clinical benefits in the treatment of patients with malignant tumors, especially lung cancer [1-3]. Although programmed death-1 (PD-1) immune checkpoint inhibitors are effective in $10 \%-20 \%$ of lung cancer patients, therapeutic effects are not observed in the rest of patients. It is thus necessary to improve the efficacy of the immune checkpoint therapy for the treatment of cancer patients who fail to respond to the current therapy. In order to modify the immune checkpoint therapy, it is indispensable to elucidate the mechanism underlying the anti-tumor effect of immune checkpoint inhibitors.

We initially established mAbs specific for PD-1 immune checkpoint molecules and demonstrated that the blockade of PD-1 signaling by anti-PD-1 ligand 1 (PD-L1) mAbs promoted the immune effector functions of killer $\mathrm{T}$ cells in animal models $[4,5]$. In 
addition, we recently reported that the anti-tumor effect of PD-1 immune checkpoint therapy was abrogated by the depletion of $\mathrm{CD} 8^{+} \mathrm{T}$ cells or natural killer (NK) cells, suggesting that not only killer T cells, but also NK cells are essential in the anti-tumor effect of the immune checkpoint inhibitors [6]. It is, therefore, crucial to examine the functions of NK cells in tumor immunity.

NK cells are innate immune cells that play pivotal roles in the first line of defense against infections and mediate anti-tumor responses [7, 8]. When pathogenic stresses are sensed by innate immune receptors, inflammasomes are assembled in the cells like macrophages and dendritic cells, resulting in the activation of caspase 1 that converts a precursor of interleukin-18 (IL-18) into mature IL-18 [9]. It is worth noting that IL-18 receptors (IL-18Rs) are highly expressed on NK cells in the peripheral blood, suggesting that NK cells are somehow regulated by IL-18 in the first line of defense against infections and malignancies. In the present study, we examined the effect of IL-18 on human NK cells and discussed the roles of NK cells in tumor immunity.

\section{Materials and Methods}

\section{Purification of peripheral blood mononuclear cells}

Peripheral blood samples $(10 \mathrm{~mL})$ were obtained from 21 healthy adult volunteers and 25 lung cancer patients after approval of the institutional review board of Nagasaki University Hospital and with written informed consent. All methods were performed in accordance with the guidelines and regulations of Nagasaki University Hospital. Peripheral blood samples were heparinized and diluted with Dulbecco's phosphate buffered saline (-) (PBS, Nissui Pharmaceutical Co., Ltd. Taito-ku, Tokyo, Japan). The diluted blood samples were loaded on Ficoll-Paque ${ }^{\mathrm{TM}}$ PLUS (GE Healthcare Bio-Sciences AB, Uppsala, Sweden) and peripheral blood mononuclear cells (PBMCs) were purified by gradient centrifugation. The cells were washed two times with PBS, then resuspended in $80 \mu \mathrm{L}$ of PBS containing $0.5 \%$ BSA fraction V (Nacalai Tesque Inc., Nakagyo-ku, Kyoto, Japan) and $2 \mathrm{mM}$ EDTA (Dojindo Laboratories, Kamimashiki-gun, Kumamoto, Japan) and the cell suspension was placed on ice.

\section{Derivation of human NK cells}

Anti-CD3 MACSBeads (20 $\mu \mathrm{L}$, Miltenyi Biotec, Auburn, CA) were added to $80 \mu \mathrm{L}$ of PBMC suspension in PBS/0.5\% BSA/2 mM EDTA and the cell suspension was placed at $4^{\circ} \mathrm{C}$. After $15 \mathrm{~min}, 2 \mathrm{~mL}$ of PBS $/ 0.5 \%$ BSA/ 2 mM EDTA were added to the cell suspension. The cell suspension was centrifuged at $300 \times \mathrm{g}$ at $4^{\circ} \mathrm{C}$ for $10 \mathrm{~min}$ and the supernatant was discarded. The cell pellets were resuspended in 0.5 $\mathrm{mL}$ of PBS/0.5\% BSA/2 mM EDTA and applied to LD Column (Miltenyi Biotec) which had been attached to a magnet holder (Miltenyi Biotec) and equilibrated with $2 \mathrm{~mL}$ of PBS/0.5\% BSA/2 mM EDTA. CD3- cells were then eluted with $2 \times 1 \mathrm{~mL}$ of PBS/0.5\% BSA/2 $\mathrm{mM}$ EDTA into a conical tube. After the tube was centrifuged at $600 \times \mathrm{g}$ for $5 \mathrm{~min}$, the supernatant was discarded and CD3- cell pellets were resuspended in 3 $\mathrm{mL}$ of Yssel's medium containing human $\mathrm{AB}$ serum (Cosmo Bio Co., Ltd., Koto-ku, Tokyo, Japan). The CD3- cell suspension was placed in two wells of a 24-well plate (Corning Inc., Corning, NY) (1.5 $\mathrm{mL} /$ well) and the plate was incubated at $37^{\circ} \mathrm{C}$ with $5 \% \mathrm{CO}_{2}$ in the presence of $100 \mathrm{U} / \mathrm{mL}$ of IL-2 (Shionogi Pharmaceutical Co., Ltd., Chuo-ku, Osaka, Japan) with or without $100 \mathrm{ng} / \mathrm{mL}$ of IL-18 (GlaxoSmithKline plc, Brenford, Middlesex, UK). In comparison with other soluble factors, CD3- cells were incubated with 100 ng/mL of IL-21 (PeproTech, Rocky Hill, NJ) and $100 \mathrm{U} / \mathrm{mL}$ of IL-2 or $100 \mathrm{ng} / \mathrm{mL}$ of IL-15 (Wako Pure Chemical Industries, Ltd., Chuo-ku, Osaka, Japan). The cytokines were added to culture media every day and complete RPMI1640 medium (Merck \& Co., Inc., Kenilworth, NJ) containing $10 \%$ fetal calf serum (FCS, Merck \& Co., Inc.), 10-5 M 2-mercaptoethanol (Nacalai Tesque Inc.), $100 \mathrm{U} / \mathrm{mL}$ penicillin (Meiji Holdings Co. Ltd., Chuo-ku, Tokyo, Japan), and $100 \mu \mathrm{g} / \mathrm{mL}$ streptomycin (Meiji Holding Co., Ltd.) was used beginning on day 5 . NK cells were harvested on day10 and analyzed for the immune effector functions and the expression of cell surface markers.

\section{Microscopic analysis}

During the derivation of NK cells, cell images were captured under a BZ-X700 fluorescence microscope (Keyence Corp., Higashiyodogawa-ku, Osaka, Japan).

\section{Tumor cell lines}

K562 (erythrocytoma), VMRC-RCW (renal cell carcinoma), Raji (Burkitt's lymphoma), RAMOS-RAI (Burkitt's lymphoma) were obtained from Health Science Research Resources Bank (Sennan, Osaka, Japan), ACHN (renal cell carcinoma) was purchased from American Type Culture Collection (Manassas, VA), and PC-9 (lung cancer) was obtained from RIKEN BioResource Center (Tsukuba, Ibaraki, Japan).

\section{Flow cytometric analysis}

PBMCs or NK cells were plated out at $2 \times 10^{5}$ cells $/ 50 \mu \mathrm{L}$ in a 96-well plate (Corning Inc.). The cells were then treated with $3 \mu \mathrm{L}$ of $\mathrm{mAbs}$, including, 
fluorescein isothiocyanate (FITC)-conjugated anticluster of differentiation 3 (CD3) and CD56 mAbs (BD Biosciences, San Diego, CA), and phycoerythrin (PE)-conjugated anti-CD16, CD25, CD56, CD80, CD86, DNAX accessory molecules-1 (DNAM-1), Fas-L, human leukocyte antigen-DQ (HLA-DQ), HLA-DR, inducible T-cell costimulator (ICOS), natural killer group 2 member D (NKG2D) and tumor necrosis factor-related apoptosis-inducing ligand (TRAIL) mAbs (BD Biosciences) on ice for $15 \mathrm{~min}$. After being washed three times with PBS, the cells were resuspended in $400 \mu \mathrm{L}$ of PBS and analyzed using a FACSCalibur flow cytometer (Becton Dickinson, Franklin Lakes, NJ).

\section{Cellular cytotoxicity assay}

NK cell-mediated cellular cytotoxicy was determined using a non-radioactive cellular cytotoxicity assay kit (Techno Suzuta, Heiwa, Nagasaki, Japan). Tumor cells $\left(1 \times 10^{6}\right.$ cells $\left./ \mathrm{mL}\right)$ were treated with $2.5 \mu \mathrm{L}$ of a precursor of a chelate-forming compound for $15 \mathrm{~min}$ at $37^{\circ} \mathrm{C}$ with $5 \% \mathrm{CO}_{2}$. After being washed three times with $5 \mathrm{~mL}$ complete RPMI1640 media, the labeled tumor cells $\left(5 \times 10^{4}\right.$ cells $/ 100 \mu \mathrm{L}$ ) in a round bottom 96-well plate (Corning Inc.) were challenged by $100 \mu \mathrm{L}$ of NK cells at effector-to-target ratios of $0,0.625,1.25,2.5,5,10,20$ and $40: 1$ for $40 \mathrm{~min}$ at $37^{\circ} \mathrm{C}$ with $5 \% \mathrm{CO}_{2}$. After being centrifuged at $600 \times \mathrm{g}$ for $2 \mathrm{~min}$, the supernatants ( 25 $\mu \mathrm{L}$ each) were removed to a new round bottom 96-well plate containing $250 \mu \mathrm{L}$ of europium solution, from which $200 \mu \mathrm{L}$ samples were transferred to a 96-well optical plate (Thermo Fisher Scientific Inc., Waltham, MA). Time-resolved fluorescence was measured through a PHERAstar FS multiplate reader (BMG Labtech Ltd., Allmendgruen, Ortenberg, Germany). All experiments were performed in triplicate. Specific lysis (\%) was calculated as $100 \mathrm{x}$ [experimental release (counts) - spontaneous release (counts)] / [maximum release (counts) - spontaneous release (counts)] and spontaneous release (\%) was calculated as100 x [spontaneous release (counts) background (counts)] / [maximum release (counts) background (counts)]. For the determination of antigen-dependent cellular cytotoxicity (ADCC), tumor cells were pre-incubated with anti-epidermal growth factor receptor (EGFR) mAb (Cetuximab, Bristol-Myers Squibb, New York, NY) at concentrations of $0,0.05$ or $0.5 \mu \mathrm{g} / \mathrm{mL}$ or with anti-CD20 mAb (Rituximab, Zenyaku Kogyo Co., Ltd., Bunkyo, Tokyo, Japan) at concentrations of 0 , 0.01 or $0.1 \mu \mathrm{g} / \mathrm{mL}$ for $15 \mathrm{~min}$ at $37^{\circ} \mathrm{C}$ with $5 \% \mathrm{CO}_{2}$.

\section{107a degranulation assay}

K562 and PC-9 cells were grown in complete
RPMI1640 media overnight. PC-9 cells $\left(1.5 \times 10^{6}\right.$ cells $/ 100 \mu \mathrm{L}$ ) were pre-incubated with 0 or $0.5 \mu \mathrm{g} / \mathrm{mL}$ of anti-EGFR mAbs for $20 \mathrm{~min}$ on ice. IL-2/IL18-induced NK cells $\left(5 \times 10^{5}\right.$ cells $\left./ 100 \mu \mathrm{L}\right)$ were incubated with K562, PC-9 or mAb-pre-treated PC-9 cells at an effector-to-target ratio of $1: 3$ at $37^{\circ} \mathrm{C}$ with $5 \% \mathrm{CO}_{2}$ in a round bottom 96-well plate (Corning Inc.). After $2 \mathrm{~h}$, the plate was centrifuged at $600 \times \mathrm{g}$ for $2 \mathrm{~min}$ at $4^{\circ} \mathrm{C}$ and the supernatant was discarded. The pellets were then resuspended in $44 \mu \mathrm{L}$ of $\mathrm{PBS} / 2 \%$ FCS, to which were added $3 \mu \mathrm{L}$ of PE-conjugated anti$\mathrm{CD} 107 \mathrm{a} \mathrm{mAb}$ (BD Biosciences) and $3 \mu \mathrm{L}$ of FITCconjugated anti-CD56 mAbs (BD Biosciences). After incubation on ice for $20 \mathrm{~min}$, the cells were washed three times with $200 \mu \mathrm{L}$ of PBS/2\% FCS, the cells were resuspeded in $500 \mu \mathrm{L}$ of PBS/2\% FCS. The degree of degranulation was determined by a FACSCalibur flow cytometer.

\section{Cytokine production}

PC-9 and VMRC-RCW cells $\left(1.5 \times 10^{6}\right.$ cells $/ 100$ $\mu \mathrm{L})$ were pre-incubated with 0 or $1 \mu \mathrm{g} / \mathrm{mL}$ of anti-EGFR mAbs for $15 \mathrm{~min}$ on ice. NK cells $\left(5 \times 10^{5}\right.$ cells/100 $\mu \mathrm{L}$ ) were incubated with PC-9, VMRCRCW, mAb-pretreated $\mathrm{K} 562$ or mAb-pretreated VMRC-RCW cells $\left(1.5 \times 10^{6}\right.$ cells $\left./ 100 \mu \mathrm{L}\right)$ at an effector-to-tumor ratio of $1: 1$ in a round bottom 96-well plate (Corning Inc.). After incubation for $5 \mathrm{~h}$ at $37{ }^{\circ} \mathrm{C}$ with $5 \% \mathrm{CO}_{2}$, the plate was centrifuged at $600 \times \mathrm{g}$ for $2 \mathrm{~min}$ and the culture supernatants were frozen at $-80^{\circ} \mathrm{C}$ overnight. The samples were then thawed and IFN- $\gamma$ levels determined by ELISA (Peprotech, Rocky Hill, NJ) according to the manufacturer's instruction.

\section{Statistical analysis}

Statistical analyses were performed in GraphPad software $t$ test calculator for paired samples. $p$ values are as given with values $<0.05$ considered significant.

\section{Results}

\section{Comparison of the proportion of NK cells in PBMCs of lung cancer patients and healthy adult volunteers}

We first analyzed the proportion of NK cells in peripheral blood lymphocytes. As shown in Supporting Information Fig. S1A, the proportions of CD3-CD56 ${ }^{+}$cells in lymphocyte gates were $19.8 \%$ and $15.3 \%$ in lung cancer patients LC-A and LC-B and $17.6 \%$ and $16.0 \%$ in healthy adult volunteers HD-A and HD-B, respectively. The average proportions of CD3-CD56 ${ }^{+}$cells were $19.2 \pm 10.5 \%$ (mean \pm SD) and $17.3 \pm 6.8 \%$ in 25 lung cancer patients and 21 healthy adult volunteers, respectively (Supporting Information Fig. S1B). The results show that the number of 
NK cells in lung cancer patients is equivalent to that in healthy volunteers $(p=0.4737)$, suggesting that NK

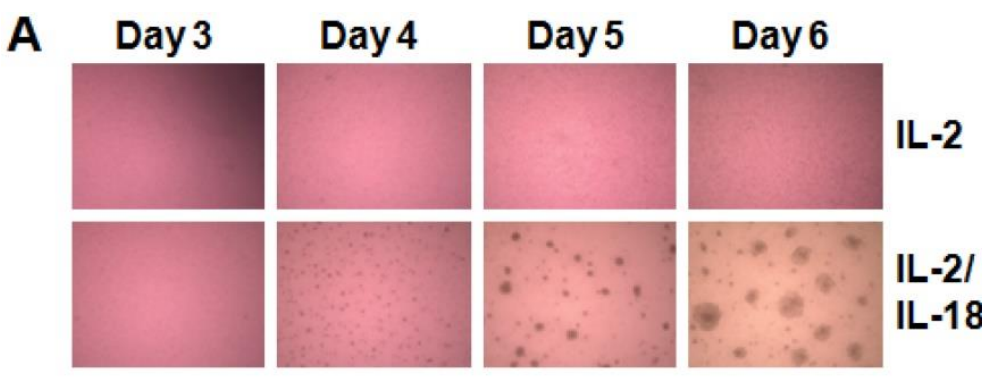

B Day 0 CD3-cells Day 10 IL-2 Day 10 IL-2/LL-18
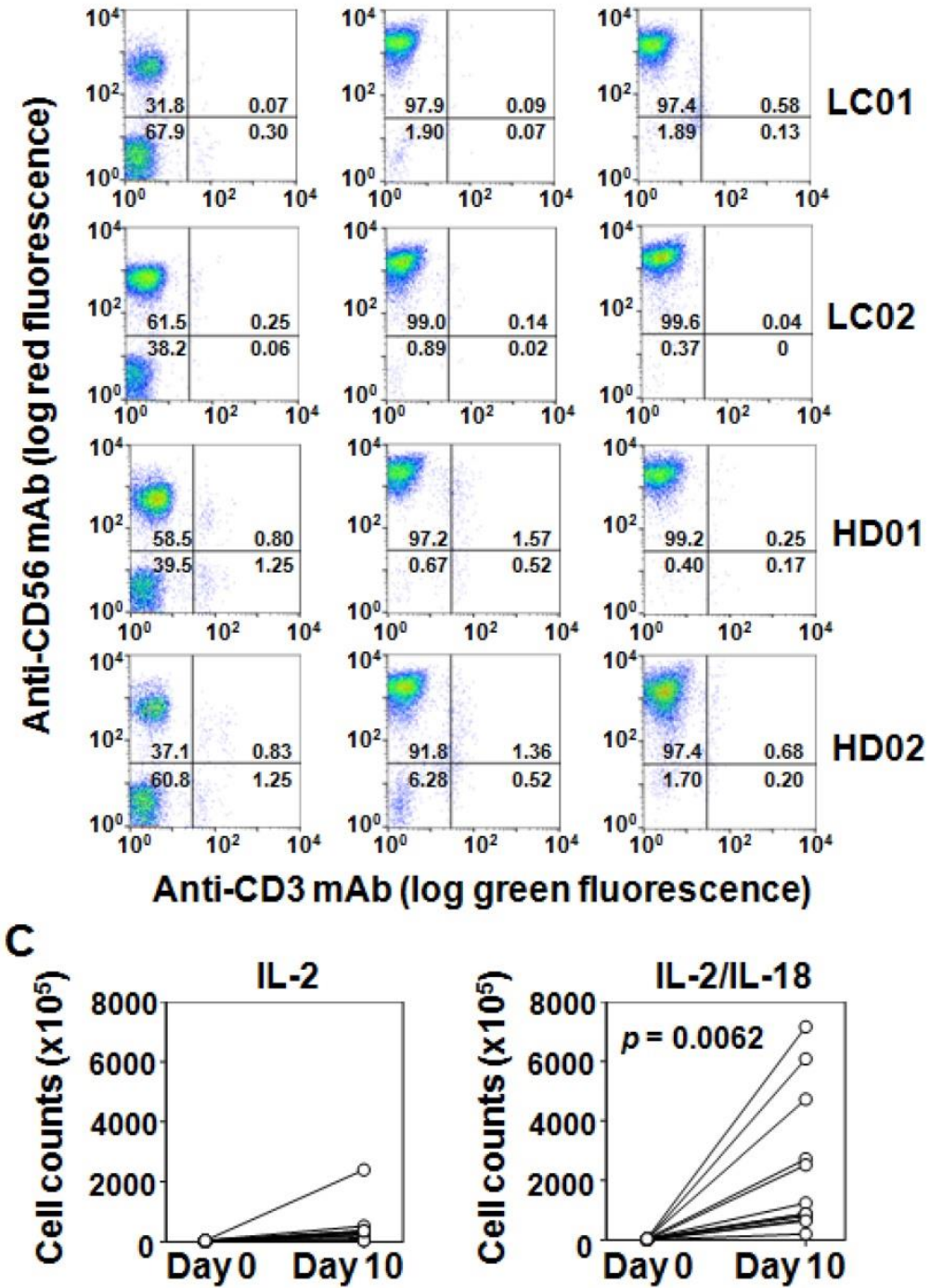

Figure 1. Effect of IL-18 on the IL-2-mediated expansion of human NK cells. (A) Microscopic analysis of the effect of IL-18 on the expansion of NK cells stimulated with IL-2. Heparinized PBMCs were obtained from a healthy adult volunteer and a lung cancer patient and CD3- PBMCs were purified by negative selection using anti-CD3 mAb-coated beads. The cell suspensions were stimulated with either IL-2 or IL-2/IL- 18 at $37 \circ \mathrm{C}$ with $5 \% \mathrm{CO}_{2}$ and cell clustering was observed under a microscope on days 3, 4, 5 and 6. (B) Flow cytometric analysis of CD3PBMCs stimulated with IL-2 or IL-2/IL-18. CD3- PBMCs derived from lung cancer patients (LC01 and LC02) and healthy donors (HD01 and HD02) were incubated with IL-2 or IL-2/IL-18 for 10 days and the expanded cells were analyzed through flow cytometry. (C) Effect of IL-18 on the number of NK cells after stimulation with IL-2. Before and after expansion of CD3- PBMCs derived from 13 lung cancer patients with IL-2 or IL-2/IL-18 for 10 days, the number of NK cells was counted by trypan blue dye exclusion. A p value between the numbers of IL-2- and IL-2/IL-18-induced NK cells is shown.

\section{Effect of IL-1 8 on the expansion of NK cells}

We next examined the effect of IL-18 on the proliferative responses of NK cells. When CD3- PBMCs derived from a lung cancer patient were stimulated with IL-2, the cells formed clusters beginning on day 5-6 and proliferated thereafter (Fig. 1A). The addition of IL-18 to the cell culture markedly enhanced the cell clustering (Fig. 1A). The cell clusters appeared as early as on day 4 and large cluster formation was observed on day 5 and day 6. Essentially the same time course of cell clustering was observed for CD3PBMCs from healthy adult volunteers (data not shown).

After 10 days of incubation with IL-2, the proportion of CD3-CD56 ${ }^{+}$cells in CD3- PBMCs derived from lung cancer patients increased from $40.5 \pm$ $16.7 \%$ on day 0 to $94.3 \pm 5.8 \%$ on day 10 . When stimulated with IL-2 and IL-18, NK cells increased up to $96.6 \pm 2.6 \%$ on day 10. The expansion profile of NK cells in CD3- cells derived from healthy adult volunteers was almost the same as that from lung cancer patients. Flow cytometric analyses of IL-2- and IL-2/IL-18-induced expansion of NK cells derived from representative lung cancer patients and healthy adult volunteers are shown in Fig. 1B.

The number of NK cells of lung cancer patients after incubation with IL-2 for 10 days increased from $4.26 \mathrm{x}$ $10^{6} \pm 1.57 \times 10^{6}$ cells on day 0 to $4.48 \times$ $10^{7} \pm 6.30 \times 10^{7}$ cells on day 10 , with the expansion rate being 10 . The addition of IL-18 promoted the expansion of NK cells up to $2.39 \times 10^{8} \pm 2.35 \times 10^{8}$ cells on day 10 , with the expansion rate being 56 as shown in Fig. 1C ( $p=0.0062)$. Essentially the same expansion profile of NK cells was obtained in CD3PBMCs derived from healthy adult volunteers, demonstrating that IL-18 promoted the expansion of NK cells and that NK cells were not functionally down-regulated in lung cancer patients. 


\section{Effect of IL-18 on the expression of cell surface markers on NK cells}

We then compared cell surface markers expressed on NK cells that had been treated with IL-2 and IL-2/IL-18. CD3- cells were first negativelyselected from PBMCs using a bead-separation column and stimulated with IL-2 or IL-2/IL-18 for 10 days and the cell surface markers were analyzed on flow cytometry. It is noteworthy that cell surface molecules typically expressed on antigen-presenting cells (APCs) such as monocytes and dendritic cells were induced on NK cells in a time-dependent manner as shown in Fig. 2. CD80, a ligand of the CD28 co-stimulatory signal receptor expressed on $\mathrm{T}$ cells, was highly induced on IL-2/IL-18-stimulated NK cells, with the mean fluorescence intensity (MFI) being 75.9 on day 10 . In contrast, the MFI of CD80 on
IL-2-stimulated NK cells was only 7.6 (Supporting Information Fig. S2). Similarly, the MFIs of CD86, another ligand of CD28, were 820 and 8.9 on day 10 for IL-2/IL-18- and IL-2-induced NK cells. In addition, the expression of HLA-DR and HLA-DQ was also induced in IL-2/IL-18-stimulated NK cells. The MFIs of HLA-DR and HLA-DQ on day 10 were 180 and 256 for IL-2/IL-18-stimulated NK cells and 11 and 13 for IL-2-stimulated NK cells, respectively. The expression of ICOS and CD25 was also higher in IL-2/IL-18-induced NK cells than that in IL-2-induced NK cells. In contrast, the expression of receptors and ligands involved in immune effector functions such as NKG2D, DNAM-1, Fas-L, TRAIL and CD16 was not significantly altered by the addition of IL-18 as shown in Supporting Information Figs. S2 and S3.

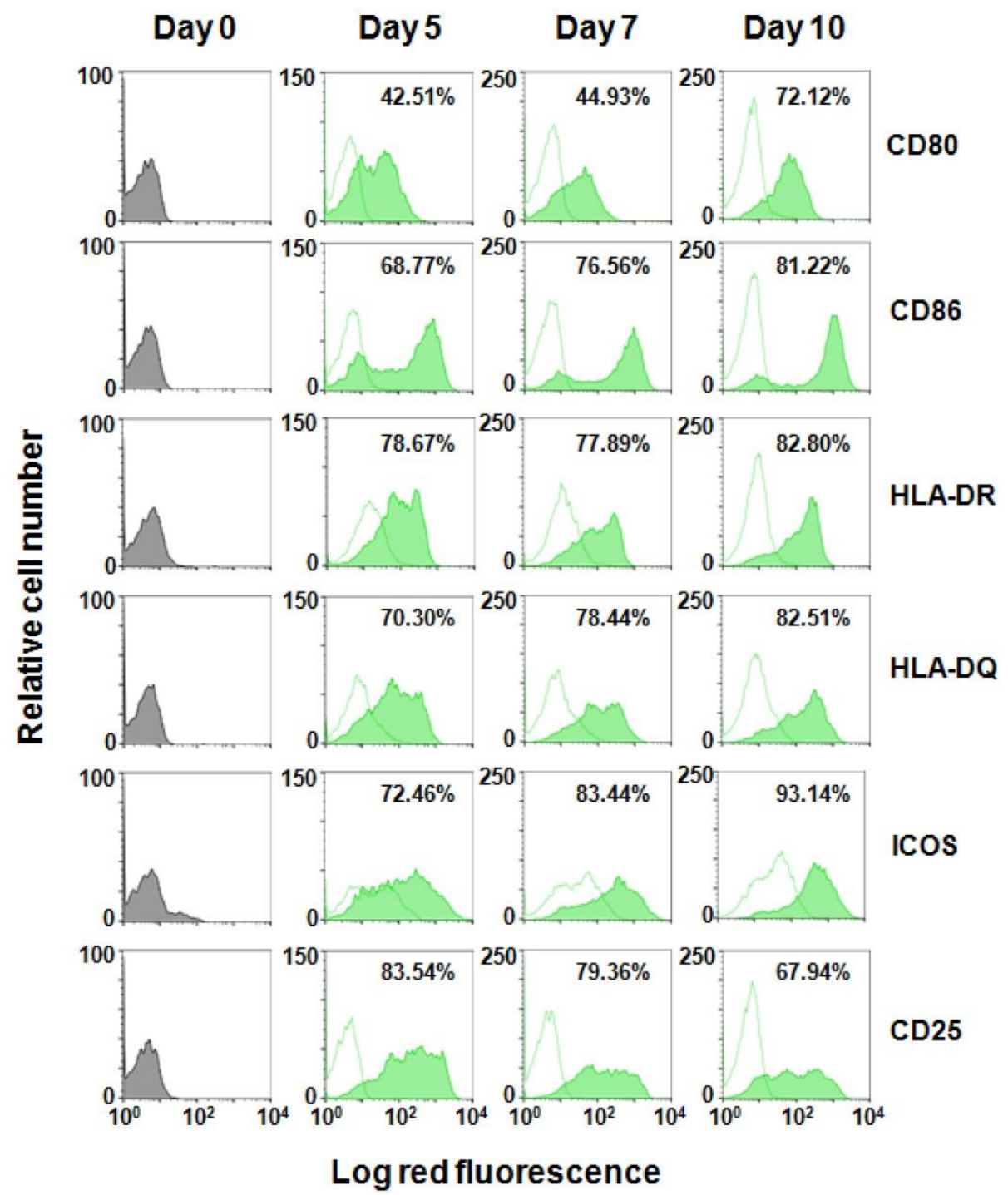

Figure 2. Effect of IL-18 on the expression of surface markers on NK cells. CD3- PBMCs derived from a healthy adult volunteer were incubated with IL-2 or IL-2/IL-18 and the expression of cell surface markers on CD56 cells were detected through flow cytometry using FITC-conjugated anti-CD56 mAb and PE-conjugated anti-CD80, CD86, HLA-DR, HLA-DQ, ICOS and CD25 mAbs on days 0, 5, 7 and 10. The flow cytometric profiles of CD56+ cells incubated with IL-2 are displayed as open histograms with dotted contours and that with IL-2/LL-18 as filled histograms with solid contours. The proportions of cell surface marker-positive cells in CD56+ cells after expansion are indicated. 
Because the IL-2/IL-18-induced NK cells expressed APC-associated molecules, we further analyzed the phenotype of $\mathrm{CD} 3-\mathrm{CD} 56^{+}$cells. It was previously reported that a subset of $\mathrm{CD} 14^{+}$monocytes in the peripheral blood had a phenotype of CD3-CD56 ${ }^{+}$[10]. We thus cultured CD3- PBMCs from a healthy adult volunteer in the presence of IL-2/IL-18 for 10 days and examined the expression of CD14. As shown in Supporting Information Fig. S4, IL-2/IL18-induced CD3-CD56 ${ }^{+}$cells were negative for CD14, whereas the expression of CD80, CD86, HLA-DR and HLA-DQ was highly positive, confirming that IL-2/IL-18-induced CD3-CD56 ${ }^{+}$cells were NK cells expressing APC-associated cell surface markers.

It is intriguing to examine whether or not other cytokines alter the phenotype of NK cells. Because IL-21 and IL-15 have been reported to regulate NK cell activation and expansion [11-16], we compared the cell surface markers of $\mathrm{CD} 3-\mathrm{CD} 56^{+}$cells incubated with IL-2, IL-2/IL-18, IL-2/IL-21 and IL-15 (Supporting Information Fig. S5). Rapid cell clustering and propagation of NK cells were observed in the culture of CD3- PBMCs with IL-2/IL-18 and the expanded NK cells expressed a high level of APC-associated molecules, CD80, CD86, HLA-DR and HLA-DQ as well as ICOS and CD25. IL-2/IL-21 induced the expression of the cell surface markers in a small subset of NK cells, whereas IL-15 had a minimal effect on their expression, demonstrating that IL-18 had a unique property in the effect on NK cells.

We next examined whether the APC-like NK cells interact with T cells. When IL-2/IL-18-induced NK cells were incubated with $\gamma \delta$ T cells, the NK cells formed clusters with $\gamma \delta \mathrm{T}$ cells, suggesting that the APC-like NK cells could functionally associate with T cells through cell-to-cell contact (Supporting Information Fig. S6).

\section{Cellular cytotoxicity exhibited by IL-2/ IL-1 8-induced NK cells}

Whereas IL-18 was likely to confer NK cells an APC-like phenotype, IL-2/IL-18-stimulated NK cells expressed a high level of immune receptors and ligands that are inexorably linked to immune effector functions. We thus examined the cellular cytotoxic activity of IL-2/IL-18-stimulated NK cells. Because NK cells kill tumor cells rapidly and effectively, a non-radioactive cellular cytotoxicity assay using a europium $(\mathrm{Eu})$ chelate was employed in this study. When K562 human erythrocytoma cells susceptible to NK cell-mediated cellular cytotoxicity were challenged by IL-2/IL-18-induced NK cells derived from a lung cancer patient, the target cells were effectively killed in an effector-to-target ratio-dependent manner, with more than $70 \%$ of K562 cells being killed within
$40 \mathrm{~min}$ at an effector-to-target ratio of $40: 1$ (Fig. 3A). When PC-9 lung cancer cells were incubated with NK cells with an APC-like phenotype, about $20 \%$ of the target cells were killed under the same condition. Because PC-9 cells expressed epidermal growth factor receptor (EGFR) as shown in Supporting Information Fig. S7, antibody-dependent cellular cytotoxicity (ADCC) was determined using anti-EGFR mAbs. As expected, the specific lysis (\%) was markedly augmented up to nearly $60 \%$ as the concentration of the $\mathrm{mAb}$ increased, indicating that the IL-2/IL-18-stimutated NK cells exhibited potent ADCC against the lung cancer cell line. When the renal cell carcinoma cell lines $\mathrm{ACHN}$ and VMRC-RCW expressing EGFR and the Burkitt's lymphoma cell lines Raji and RAMOS-RAI expressing CD20 (Supporting Information Fig. S7) were used as target cells, the IL-2/IL-18-induced NK cells killed them efficiently and the specific lysis (\%) was increased by the addition of anti-EGFR $\mathrm{mAb}$ or anti-CD20 $\mathrm{mAb}$ in a mAb-dose dependent manner (Fig. 3A). The cytotoxic activity against tumor cells of the IL-2/L-18-stimulated NK cells derived from lung cancer patients were equivalent to that derived from healthy adult volunteers (Fig. 3B). In order to confirm the cellular cytotoxicity, the degree of degranulation of IL-2/IL-18-induced NK cells was determined by flow cytometry. As shown in Fig. 4A, degranulation was observed in the APC-like NK cells when incubated with PC-9 and K562 cells and the degree of degranulation was increased when PC-9 cells were preincubated with anti-EGFR mAbs. The results demonstrate that NK cells with an APC-phenotype exhibit conventional NK activity as well as ADCC activity.

\section{IFN- $\gamma$ production by IL-2/IL-1 8-induced NK cells in response to tumor cells}

To further examine effector functions, IL-2/IL-18-stimulated NK cells derived from lung cancer patients and healthy adult volunteers were challenged by PC-9 and VMRC-RCW cells at an effector-to-target ratio of $1: 1$ for $5 \mathrm{~h}$ (Fig. 4B). IL-2/IL-18-stimulated NK cells derived from lung cancer patients secreted IFN- $\gamma$ in response to tumor cell lines and the production of the cytokine was equivalent to that from healthy adult volunteers. In addition, the cytokine production was augmented when the tumor cell lines were pretreated with anti-EGFR mAbs. Collectively, IL-2/IL-18-induced human NK cells with an APC phenotype exhibit effector functions including conventional cellular cytotoxicity, ADCC and cytokine production. 


\section{Discussion}

The clinical success of immune checkpoint inhibitors has propelled the development of novel cancer immunotherapies [17]. Although PD-1 immune checkpoint inhibitors have been approved as
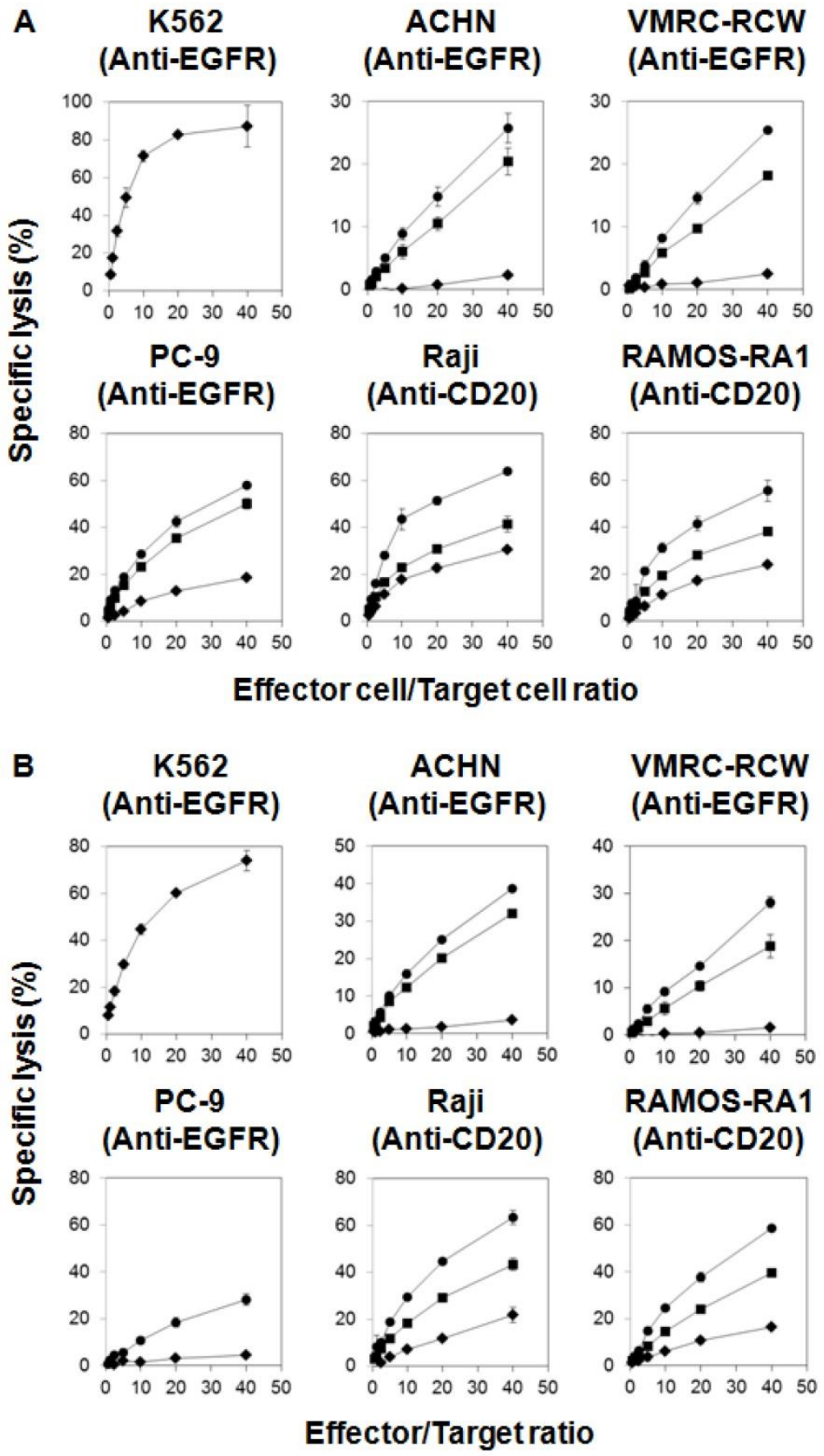

Figure 3. Cellular cytotoxicity and ADCC against tumor cell lines exhibited by IL-2/IL-18-stimulated NK cells. CD3- PBMCs derived from a lung cancer patient (A) and a healthy adult volunteer (B) were stimulated with IL-2/IL-18 for 10 days and cellular cytotoxicity and ADCC against K562, PC-9, ACHN, VMRC-RCW, Raji and RAMOS-RAl exhibited by NK cells were determined using a non-radioactive cellular cytotoxicity assay kit. PC-9, ACHN and VMRC-RCW were pretreated with anti-EGFR mAb at concentrations of $0(\bullet), 0.05(\boldsymbol{\bullet})$ or $0.5 \mu \mathrm{g} / \mathrm{mL}(\bullet)$ and Raji and RAMOS-RAI with anti-CD20 mAb at concentrations of $0(\bullet), 0.01(\bullet)$ or $0.1 \mu \mathrm{g} / \mathrm{mL}(\bullet)$ at $37 \circ \mathrm{C}$ with $5 \% \mathrm{CO}_{2}$ for $15 \mathrm{~min}$. The spontaneous release (\%) was less than $20 \%$ in all the experiments. All experiments were done in triplicate. therapeutics for the treatment of patients with malignancies, the immunotherapy is effective for only a subset of cancer patients. It is thus indispensable to improve the current immunotherapy by combination with other modalities.

Recently, we found that NK cells played an essential role in a combination immune checkpoint therapy in an animal model [6]. Because CD8 ${ }^{+}$killer $\mathrm{T}$ cells are generally considered to be immune effector cells responsible for the eradication of tumor cells, the mechanism underlying the anti-tumor effect of NK cells in PD-1 immune checkpoint therapy is obscure. We, therefore, set out to analyze the functions of NK cells in tumor immunity.

NK cells belong to the innate immune system and play an essential role in host immunity against cancer as well as infections [18]. In the first line of defense, pathogenic stresses are first sensed by pattern recognition receptors like Toll-like receptors on innate immune cells such as macrophages and dendritic cells, and inflammasomes in the cytoplasm are activated. Caspase 1, a component of inflammasomes, then converts a precursor of IL-18 to its mature form that is secreted from the innate immune cells $[19,20]$. Because NK cells express a high level of IL-18Rs, we examined the effect of IL-18 on the functions and phenotype of human NK cells in this study.

The most prominent effect of IL-18 was the acceleration in the IL-2-induced expansion of human NK cells, consisting with the reports demonstrating that the IL-2-mediated expansion of mouse NK cells was enhanced by the addition of IL-18 [21]. NK cell-based immunotherapy has attracted much attention in the treatment of high-risk cancer patients. It is, however, still a challenge to prepare large numbers of good manufacturing practice (GMP)-grade NK cells. Although depletion of $\mathrm{CD}^{+}$ cells from PBMCs followed by enrichment of $\mathrm{CD}^{+} 6^{+}$cells using CliniMACS instruments results in a good recovery of CD3-CD56 ${ }^{+}$cells, NK cells expand moderately in response to IL-2 to a median fourfold ranging from twofold to eightfold in the majority of PMBCs 
derived from healthy donors [22, 23]. Feeder cells seem to promote the expansion of NK cells effectively. For instance, NK cells expanded 490-fold in 21 days in response to irradiated Epstein-Barr virus-transformed lymphoblastoid cells plus IL-2 [24] and 758-fold in 14 days when stimulated with irradiated autologous PBMCs, anti-CD3 mAbs and IL-2 [25].

In addition to IL-2, other cytokines such as IL-21 and IL-15 have been shown to affect NK cell functions [11-16]. The soluble cytokines, however, fail to support the expansion of NK cells as shown in Supplementary Information Fig. S5. In contrast, membrane-bound IL-15 and IL-21 have been reported to efficiently expand NK cells ex vivo [26-30]. For instance, genetically-modified K562 cells expressing 4-1BBL and membrane-bound IL-15 induced 1,000-fold expansion of NK cells in 21 days [31], and those expressing membrane-bound IL-21 resulted in 21,000-fold expansion [32]. The drawback of the feeder cell-supported expansion of NK cells is a difficulty in efficient removal of feeder cells in standard GMP facilities. To overcome the problem, immobilized 4-1BBL and IL-21-coated beads were developed, in which 140-fold expansion of NK cells were reported [33]. Because IL-18 not only augments the proliferation of $\mathrm{NK}$ cells, but also change the phenotype of NK cells into that of APC-like cells, IL-18 may be useful for the preparation of GMP-grade NK cells for a novel adjuvant immunotherapy. Many clinical trials are now underway to establish novel cancer immunotherapies in combination with other modalities including adoptive transfer of immune effector cells and IL-18 is a useful measure to effectively expand NK cells ex vivo.

Previously, we found that APC-associated molecules such as CD80, CD86, HLA-DR and HLA-DQ were expressed on immune effector cells, when PMBCs were stimulated with a nitrogencontaining bisphosphonate, IL-2 and IL-18 in an aim at expanding $\gamma \delta \mathrm{T}$ cells [34]. In our report, the direct effect of IL-18 on NK cells was not clearly shown, because a mixed cell culture system was employed. In this study, we used a simple culture system to simulate NK cells with IL-2 and IL-18 and then demonstrated that IL-18 played an essential role in the expression of APC-associated molecules on NK cells. Although it is not evident that the IL-2/IL-18-induced NK cells with an APC-like phenotype have a capacity to present antigenic peptides to $\alpha \beta$ T cells, it is clear that IL-2/IL-18-induced NK cells avidly interact with $\mathrm{T}$ cells and help $\mathrm{T}$ cells to proliferate efficiently, in which cell-to-cell contact, rather than soluble factors, is essential $[35,36]$. Based on the findings, it is most likely that IL-18 confers NK cells an APC-like phenotype as well as functions.

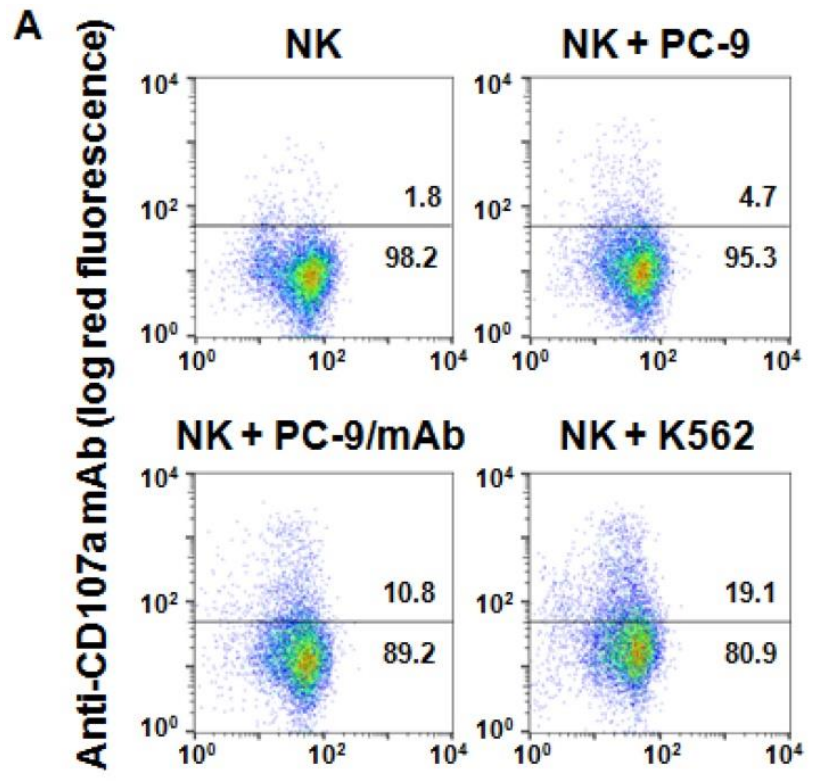

Anti-CD56 mAb (log green fluorescence)

B
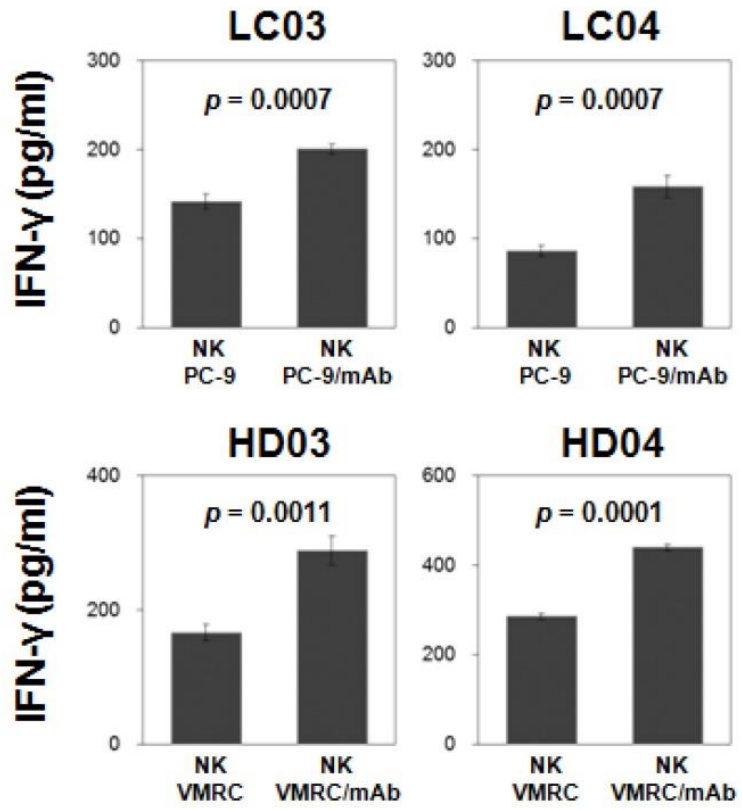

Figure 4. Effector functions exhibited by IL-2/IL-18-induced NK cells. (A) CD107a degranulation assay in IL-2/IL-18-induced NK cells in response to tumor cell lines. PC-9 cells were preincubated with 0 or 0.5 $\mu \mathrm{g} / \mathrm{mL}$ of anti-EGFR $\mathrm{mAbs}$ for $20 \mathrm{~min}$ on ice. IL-2/LL-18-stimulated NK cells from an adult donor were incubated with PC-9 or K562 cells at an effector-to-target ratio of $1: 3$ and the degree of degranulation was analyzed by flow cytometry using PE-conjugated anti-CD 107a mAbs. The experiments were done in triplicate and a representative dot plot is shown. (B) IFN- $\gamma$ production from IL-2/IL-18-induced NK cells in response to tumor cells. PC-9 cells and VMRC-RCW cells were preincubated with 0 or $1 \mu \mathrm{g} / \mathrm{mL}$ of anti-EGFR mAbs for $15 \mathrm{~min}$ on ice. IL-2/IL-18-stimulated NK cells were incubated with PC-9 or VMRC-RCW cells at an effector-to-tumor ratio of $1: 1$ and IFN- $\gamma$ production was measured through a flow cytometer using PE-conjugated anti-IFN- $\gamma$ mAb. The experiments were done in triplicate and $p$ values between IFN- $\gamma$ productions in the absence and presence of $m A b$ are shown. 
Because IL-2/IL-18-induced NK cells expressed APC-associated cell surface markers, we next examined whether the APC-like NK cells retained the cytotoxic activity against tumor cells. To determine cytotoxicity mediated by immune effector cells, $\left.{ }^{51} \mathrm{Cr}\right]-$ release assay has been generally used [37]. In the standard $\left[{ }^{51} \mathrm{Cr}\right]$-release assay, $\left[{ }^{51} \mathrm{Cr}\right]$ sodium chromate permeates into the cytoplasm of target tumor cells and binds to intracellular proteins. The protein-bound $\left[{ }^{51} \mathrm{Cr}\right]$ sodium chromate no longer diffuses out of the cells. When the labeled cells are challenged by immune effector cells, the integration of tumor cell membrane is disrupted. During the initial phase of the membrane disruption, however, the protein-bound $\left[{ }^{51} \mathrm{Cr}\right]$ sodium chromate would not diffuse out of the cells, because the size of the membrane hall is too small for the complex to pass through. Thus, it takes $2 \sim 4 \mathrm{~h}$ to determine cellular cytotoxicity using the standard $\left[{ }^{51} \mathrm{Cr}\right]$-release assay system.

In this study, we used a non-radioactive cellular cytotoxicty assay protocol to determine the specific lysis of tumor cells by the APC-like NK cells. In this assay system, target tumor cells are first treated with a precursor of chelate-forming compound with a low molecular weight. Once the precursor is internalized into tumor cells, intracellular esterases hydrolyze it to yield a negatively-charged chelate-forming substance. When the labeled tumor cells are killed by NK cells, the compound is released from the tumor cells into culture media. The chelate-forming compound forms a complex with europium and the resulting chelate complex emits long-lived fluorescence upon excitation with laser pulses, by which the cellular cytotoxicity can be quantitatively determined. Because the small chelate-forming substance generated in the cells does not interact avidly with the intracellular proteins, it rapidly diffuses out of cells when the integrity of the cell membrane is disrupted. In this assay system, it takes only 20 40 min to complete the release of the chelate when tumor cells are challenged by NK cells.

The non-radioactive assay system revealed that the APC-like NK cells killed more than $80 \%$ of K562 cells in $40 \mathrm{~min}$ at an effector-to-target ratio of $40: 1$, demonstrating that the IL-2/IL-18-induced NK cells had a potent cytotoxic activity against tumor cells. Furthermore, the APC-like NK cells derived from lung cancer patients exhibited potent anti-tumor activity against the lung cancer cell line that had been treated with anti-EGFR $\mathrm{mAb}$, indicating that IL-2/IL-18-induced NK cells showed potent ADCC activity in a CD16-dependent manner. The cytotoxic activity of the APC-like NK cells was also confirmed by the standard degranulation assay, which suggested that the killing activity was through granule-mediated exocytosis by NK cells. In addition, the IL-2/IL-18-induced NK cells secreted IFN- $\gamma$ in response to tumor cells, which also confirmed that the APC-like NK cells had conventional cytokinemediated anti-tumor activity.

Taken together, IL-2/IL-18-induced NK cells with an APC-like phenotype not only kill tumor cells through conventional NK-associated receptors and ligands such as NKG2D, DNAM-1 and CD16, but also promote the proliferation of tumor-specific killer $\mathrm{T}$ cells $[36,38]$. If this is the case, APC-like NK cells serve as a bridge between innate immunity and adaptive immunity, by which the IL-2/IL-18-induced NK cells may enhance the anti-tumor effect in PD-1 immune checkpoint therapy. Thus, it is plausible to hypothesize that the infusion of NK cells ex vivo-expanded with IL-2 and IL-18 is beneficial to lung cancer patients undergoing PD-1 immune checkpoint therapy.

\section{Abbreviations}

DNAM-1: DNAX accessory molecules-1; EGFR: epidermal growth factor receptor; HLA: human leukocyte antigen; ICOS: inducible T-cell costimulator; NKG2D: natural killer group 2 member D; PBMCs: peripheral blood mononuclear cells; PD-1: programmed death-1; TRAIL: tumor necrosis factor-related apoptosis-inducing ligand.

\section{Supplementary Material}

Supplementary figures.

http://www.ijbs.com/v14p0331s1.pdf

\section{Acknowledgments}

Recombinant human IL-18 was a kind gift from GlaxoSmithKline plc, Brenford, Middlesex, UK. This work was supported by Grants-in-Aid for Scientific Research from the Ministry of Education, Science, Culture, Sports, and Technology of Japan (MEXT) (16K08844 to YT) by Grants-in-Aid for Scientific Research from Japan Agency for Medical Research and Development (DNW-17004 and A90 to YT) and by Grants-in-Aid for Scientific Research from Translational Research Informatics Center (2017 to YT).

\section{Ethics Committee Approval and Patient Consent}

Peripheral blood samples were obtained from lung cancer patients after approval of the institutional review board of Nagasaki University Hospital and with written informed consent.

\section{Competing Interests}

YT is a co-inventor of Japanese Patent 2014-73475 
on the development of a non-radioactive cellular cytotoxicity assay using a precursor of a novel Eu chelate-forming compound. The other authors have no conflicts of interest.

\section{References}

1. Topalian SL, Drake CG, Pardoll DM. Immune checkpoint blockade: a common denominator approach to cancer therapy. Cancer Cell. 2015; 27: 450-61.

2. Topalian SL, Hodi FS, Brahmer JR, Gettinger SN, Smith DC, McDermott DF, et al. Safety, activity, and immune correlates of anti-PD-1 antibody in cancer. N Engl J Med. 2012; 366: 2443-54.

3. Brahmer JR, Tykodi SS, Chow LQ, Hwu WJ, Topalian SL, Hwu P, et al. Safety and activity of anti-PD-L1 antibody in patients with advanced cancer. N Engl J Med. 2012; 366: 2455-65.

4. Ishida M, Iwai Y, Tanaka Y, Okazaki T, Freeman GJ, Minato N, et al. Differential expression of PD-L1 and PD-L2, ligands for an inhibitory receptor PD-1, in the cells of lymphohematopoietic tissues. Immunol Lett. 2002; 84: $57-62$

5. Iwai $\mathrm{Y}$, Ishida M, Tanaka $\mathrm{Y}$, Okazaki T, Honjo T, Minato N. Involvement of PD-L1 on tumor cells in the escape from host immune system and tumor immunotherapy by PD-L1 blockade. Proc Natl Acad Sci USA. 2002; 99: 12293-7.

6. Ma Z, Li W, Yoshiya S, Xu Y, Hata M, El-Darawish Y, et al. Augmentation of Immune Checkpoint Cancer Immunotherapy with IL18. Clin Cancer Res. 2016; 22: 2969-80.

7. Moretta A, Bottino C, Vitale M, Pende D, Cantoni C, Mingari MC, et al. Activating receptors and coreceptors involved in human natural killer cell-mediated cytolysis. Annu Rev Immunol. 2001; 19:197-223.

8. Lanier LL. NK cell recognition. Annu Rev Immunol. 2005; 23: 225-74.

9. Davis $\mathrm{BK}$, Wen $\mathrm{H}$, Ting JP. The inflammasome NLRs in immunity, inflammation, and associated diseases. Annu Rev Immunol. 2011; 29: 707-35.

10. Sconocchia G, Keyvanfar K, Ouriaghli EF, Grube M, Rezvani K, Fujiwara H, et al. Phenotype and function of a CD56 ${ }^{+}$peripheral blood monocyte. Leukemia. 2005; 19: 69-76.

11. Parrish-Novak J, Dillon SR, Nelson A, Hammond A, Sprecher C, Gross JA, et al. Interleukin 21 and its receptor are involved in NK cell expansion and regulation of lymphocyte function. Nature. 2000; 408: 57-63.

12. Kasaian MT, Whitters MJ, Carter LL, Lowe LD, Jussif JM, Deng B, et al. IL-21 limits NK cell responses and promotes antigen-specific $\mathrm{T}$ cell activation: A mediator of the transition from innate to adaptive immunity. Immunity. 2002; 16: 559-569.

13. Wendt K, Wilk E, Buyny S, Schimidt RE, Jacobs R. Interleukin-21 differentially affects human natural killer cell subsets. Immunology. 2007; 122: 486-495.

14. Li Q, Y L-J, Ren H-L, Huyan T, Li J, Shi JL, et al. Multiple effects of IL-21 on human NK cells in ex vivo expansion. Immunobiology. 2015; 220: 876-888.

15. Carson WE, Giri JG, Lindenmann MJ, Linett ML, Ahdieh M, Paxton R, et al. Interleukin (IL) 15 is a novel cytokine that activates human natural killer cells via components of the IL-2 receptor. J Exp Med. 1994; 180: 1395-1403.

16. Carson WE, Fehniger TA, Haldar S, Eckhert K, Lindemann MJ, Lai C-F, et al. A potential role for interleukin-15 in the regulation of human natural killer cell survival. J Clin Invest. 1997; 99: 937-943.

17. Philips GK, Atkins M. Therapeutic uses of anti-PD-1 and anti-PD-L1 antibodies. Int Immunol. 2015; 27: 39-46.

18. Cerwenka A, Lanier LL. Natural killer cell memory in infection, inflammation and cancer. Nat Rev Immunol. 2016; 16: 112-23.

19. Akira S, Takeda K. Toll-like receptor signalling. Nat Rev Immunol. 2004; 4: 199-511.

20. Arend WP, Palmer G, Gabay C. IL-1, IL-18, and IL-33 families of cytokines. Immunol Rev. 2008; 223: 20-38.

21. Tomura M, Zhou XY, Maruo S, Ahn HJ, Hamaoka T, Okamura H, et al. A critical role for IL-18 in the proliferation and activation of NK1.1+ CD3- cells. J Immunol. 1998; 160: 4738-46.

22. Koehl U, Soerensen J, Esser R, Zimmermann S, Gruettner HP, Tonn T, et al. IL-2 activated NK cell immunotherapy of three children after haploidentical stem cell transplantation. Blood Cells Mol Dis. 2004; 33: 261-266.

23. Koehl U, Brehm C, Huenecke S, Zimmermann SY, Kloess S, Bremm M, et al. Clinical grade purification and expansion of NK cell products for an optimized manufacturing protocol. Front Oncol. 2013; 3:118. doi: 10.3389/fonc.2013.00118

24. Berg M, Lundqvist A, McCoy PJ, Samsel L, Fan Y, Tawab A, et al. Clinical grade ex vivo-expanded human natural killer cells upregulate activating receptors and death receptor ligands and have enhanced cytolitic activity against tumor cells. Cytotherapy. 2009; 11: 341-355.

25. Yang Y, Lim O, Kim TM, Ahn Y-O, Choi H, Chung H, et al. Phase I study of random healthy donor-derived allogeneic natural killer cell therapy in patients with malignant lymphoma or advanced solid tumors. Cancer Immunol Res. 2016; 4: 215-224.

26. Farhan S, Lee DA, Champlin RE, Ciurea SO, NK cell therapy: targeting disease relapse after hematopoietic stem cell transplantation. Immunotherapy. 2012; 4: 305-313.
27. Fujisaki H, Kakuda H, Shimasaki N, Imai C, Ma J, Lockey T, et al. Expansion of highly cytotoxic human natural killer cells for cancer therapy. Cancer Res. 2009; 69: 4010-4017.

28. Shah NN, Baird K, Delbrook CP, Fleisher TA, Kohler ME, Rampertaap S, et al. Acute GVHD in patients receiving IL-15/4-1BBL activated NK cells following T-cell-depleted stem cell transplantation. Blood. 2015; 125: 784-792.

29. Denman CI, Senyukov VV, Somanchi SS, Phatarpekar PV, Kopp LM, Johnson JL, et al. Membrane-bound IL-21 promotes sustained ex vivo proliferation of human natural killer cells. PLOSone. 2012; 7: e30264.

30. Ciurea SO, Schafer JR, Bassett R, Denman CJ, Cao K, Willis D, et al. Phase I clinical trial using mbIL-21 ex vivo-expanded donor-derived NK cells after haploidentical transplantation. Blood. 2017; 130: 1857-1868.

31. Imai C, Iwamoto S, Campana D. Genetic modification of primary natural killer cells overcomes inhibitory signals and induces specific killing of leukemic cells. Blood. 2005; 106: 376-383.

32. Somanchi SS, Senyukov VV, Denman CJ, Lee DA. Expansion, purification, and functional assessment of human peripheral blood NK cells. J Vis Exp. 2011; doi: $10.3791 / 2540$

33. Li XM, He CH, Liu CZ, Ma J, Ma P, Cui HL, et al. Expansion of NK cells from PBMCs using immobilized 4-1BBL and interleukin-21. Int J Oncol. 2015; 47: 335-342

34. Sugie T, Murata-Hirai K, Iwasaki M, Morita CT, Li W, Okamura H, et al. Zoledronic acid-induced expansion of $\gamma \delta \mathrm{T}$ cells from early-stage breast cancer patients: effect of IL-18 on helper NK cells. Cancer Immunol Immunother. 2013; 62: 677-87.

35. Tsuda J, Li W, Yamanishi H, Yamamoto H, Okuda A, Kubo S, et al. Involvement of CD56brightCD11ct cells in IL-18-mediated expansion of human $\gamma \delta$ T cells. J Immunol. 2011; 186: 2003-12.

36. Mailliard RB, Alber SM, Shen H, Watkins SC, Kirkwood JM, Herberman RB, et al. IL-18-induced CD83+CCR7+ NK helper cells. J Exp Med. 2005; 202: 941-53.

37. Brunner KT, Mauel J, Cerottini JC, Chapuis B. Quantitative assay of the lytic action of immune lymphoid cells on ${ }^{51} \mathrm{Cr}$-labelled allogeneic target cells in vitro; inhibition by isoantibody and by drugs. Immunology. 1968; 14: 181-196.

38. Kalinski P, Giermasz A, Nakamura Y, Basse P, Storkus WJ, Kirkwood JM, et al. Helper role of NK cells during the induction of anticancer responses by dendritic cells. Mol Immunol. 2005; 42: 535-9. 